\title{
Anti-inflammation and anti-apoptosis effects of pearl extract gel on UVB irradiation $\mathrm{HaCaT}$ cells
}

\author{
Yueh-Lung Yang ${ }^{\mathrm{a}}$, Chin-Hsien Chang ${ }^{\mathrm{b}, \mathrm{c}}$, Ching-Cheng Huang ${ }^{\mathrm{d}}$ and Hsia-Wei Liu ${ }^{\mathrm{e}, *}$ \\ ${ }^{a}$ Department of Traditional Chinese Medicine, Chang Gung Memorial Hospital, Keelung, Taiwan \\ ${ }^{b}$ Department of Chinese Medicine, En Chu Kong Hospital, New Taipei City, Taiwan \\ ${ }^{c}$ Department of Cosmetic Science, Chang Gung University of Science and Technology, Taoyuan, \\ Taiwan \\ ${ }^{d}$ Department of Biomedical Engineering, Ming-Chuan University, Taoyuan, Taiwan \\ ${ }^{e}$ Department of Life Science, Fu Jen Catholic University, New Taipei City, Taiwan
}

\begin{abstract}
Caused by acute radiation skin reaction and injury, receiving radiotherapy treatment process is often performed side-effects on cancer patients. The clinical manifestations of skin irritation, itching, peeling, pigmentation, ulcer bleeding and other symptoms, in addition to causing patient discomfort and affecting quality of life, may increase the risk of local or systemic infection, and lead to interruption of radiation therapy. At present, for acute radiation dermatitis, there is no uniform treatment, and the various methods are evaluated variously. In this study, the authors focus on broken pearls using room temperature super extraction system, the water extraction process of wet-grinding method, nano-scale pearl, along with a large number of high purity natural amino acid extracts in the water. The room-temperature super-extraction system (RTSES) can be extracted from a relatively high-volume of pearl extract. We use pearl extract as the main component of experimental material, and the blending of pearl extract and poly ( $\gamma$-glutamic acid) is used to form biodegradable composite hydrogels. This study aims to evaluate the use of RTSES to extract the major active components of pearl and enhance their antiinflammation and anti-apoptosis effects. The possible effect of pearl extract on inducing apoptosis in human keratinocyte cells $(\mathrm{HaCaT})$ under the exposure of low dose UVB has been investigated. Various concentrations of pearl extracts have been used to study the effect of low dosage UVB on $\mathrm{HaCaT}$ cells. The results show that pearl extract has no toxic effect on $\mathrm{HaCaT}$ cells. Combining the pearl extract and poly ( $\gamma$-glutamic acid) hydrogels with UVB irradiation would decrease the inflammation and apoptosis of $\mathrm{HaCaT}$ cells. The commercial pearl extract has the potential to inhibit radiation dermatitis occurring within keratinocyte cells.
\end{abstract}

Keywords: pearl extract, poly ( $\gamma$-glutamic acid), UVB irradiation, anti-inflammation, anti-apoptosis

Yueh-Lung Yang, Chin-Hsien Chang contributed equally to this work and share the first authorship.

* Address for correspondence: Hsia-Wei Liu, Department of Life Science, Fu Jen Catholic University, No. 510, Jhongiheng Rd., Sinjhuang Dist., New Taipei City 24205, Taiwan. Tel.: +886-2-29053740; Fax: +886-2-29052193; E-mail: 079336@mail.fju.edu.tw.

0959-2989/15/\$35.00 @ 2015 - IOS Press and the authors. 


\section{Introduction}

Pearl, which is composed of nacre, is produced in an active physiological environment by molluscs. Pearl, nacre, and bone are all bio-mineralization products of organisms. Pearl and nacre are basically composed of calcium carbonate, whereas bone mainly consists of calcium phosphate or hydroxyapatite (HA). Although their components are different, parts of the complex machineries that direct their formation could be homologous. Pearl powder, which could be employed as a traditional Chinese medicine, has been used to treat palpitations, insomnia, convulsions, epilepsy, and ulcers for a long history. Clinical applications of pearl powder could prove obvious therapeutic efficacy in treating relapsed aphthous ulcer, gastric ulcer, and duodenal ulcer [1]. Scallop shell extract can protect keratinocyte cells from UV-B-induced damage in vitro by acting as an antioxidant and a growth promoter. To establish the growth activity of scallop shell extract, the effect is investigated in vivo. Therefore, exposure of rat dorsal skin to UV-B is employed to produce erythema and eschar. Also, the pearl extract is observed to act as a factor to growth and cell proliferation in the previous works $[2,3]$. In recent years, there have been several studies on the cloning of the shell organic matrix proteins [46]. The shell organic matrix proteins are composed of two kinds of $\mathrm{CaCO}_{3}$ polymorphs of the primatic layer and the nacreous layer. The nacreous layer (pearl) has been used in Chinese medicine since ancient times for keeping the skin moist. The nacreous layer has also been widely used as a cosmetic in Japan. However, only few reports on the bio-active substances in the shell have been found [7-9]. Furthermore, the room-temperature super extraction system (RTSES) is a new, organic and solventfree extraction technology for ultrasonic extraction of molecules from solid particles [10]. The roomtemperature super extraction system contains an ultrasound-producing element that provides concentrated ultrasonic waves, and acoustic cavities that crush the particles in the fluid. As a result, relative large molecules in the extract retain their activities, and no organic waste and contaminants are present in the extract [11]. Particularly, the room-temperature super extraction system (RTSES) may have a higher extraction yield, lower extraction temperature, shorter extraction time and less power consumption, and preserve the extracts' bio-activities, making it suitable for the extraction of pearl powder components. Poly ( $\gamma$-glutamic acid) $(\gamma$-PGA) is a natural edible polypeptide in which glutamate is polymerized via gamma-amide linkages. Previous studies have found that $\gamma$-PGA promoted corneal wound healing and showed similar effects on hyaluronic acid [12]. These results indicate that $\gamma$-PGA can stimulate corneal wound healing by an anti-inflammatory effect and enhance cell migration and cell proliferation. $\gamma$-PGA is a promising bio-material that may be a substitute for hyaluronic acid in corneal wound healing treatment. In this study, the possible effect of pearl extract on inducing apoptosis in human keratinocyte cells $(\mathrm{HaCaT})$ under the exposure of low dose UVB is investigated as the focus. Various concentrations of pearl extracts have been used to study the effect of low dosage UVB on HaCaT cells. Cytotoxicity of pearl extract and $\gamma$-PGA composite hydrogels on HaCaT and L929 cells is determined by using MTT assay. Meanwhile, the expressions of apoptotic inflammatory mediators and inflammation-related genes are measured through real-time PCR at transcriptional and translational levels respectively.

\section{Material and method}

\subsection{Reagents}

Dulbecco's modified Eagle's medium (DMEM), fetal bovine serum (FBS), penicillin, streptomycin 
and amphotericin (PSA) were purchased from Gibco. MTT, DMSO and RIPA buffer were purchased from Sigma-Aldrich. TRIzol reagent was purchased from Ambion. Qubit® Protein Assay Kit and Qubit $^{\circledR}$ Protein Assay Kit were purchased from Invitrogen. Power SYBR green reagent and cDNA reverse transcription kits were purchased from ABI. Human IL-6 and TNF-alpha screening set were purchased from Thermo.

\subsection{Preparation of pearl extract using RTSES}

For the room-temperature super-extraction system procedure, $1 \mathrm{~kg}$ of pearl crude powder was employed and suspended in $10 \mathrm{~L}$ of deionized water and extracted by the room-temperature super-extraction system with an extraction temperature of $25^{\circ} \mathrm{C}$, extraction frequency of 40 $\mathrm{kHz}$, and extraction time of $0.5 \mathrm{~h}$. After the extraction, the size of residual powder particles was determined and observed to be 70-300 nm using a laser particle-size analyzer (Zetasizer Nano ZS; Malvern Instruments, UK). The extracted solutions (pearl extract) were vacuum freeze-dried. The vacuum freeze-drying process was free from impurities, and could maintain the original components and active ingredients without destruction of cells or material shrinkage. The dried powders were additionally dissolved in distilled water, and the impurity-free solutions were stored at $-80^{\circ} \mathrm{C}$ until use. The working concentration was determined by the initial weight of the raw materials $(\mathrm{g})$ and the final vehicle volume $(\mathrm{mL})$ after vacuum freeze-drying.

\subsection{Cell Culture}

Human keratinocyte HaCaT cells and mouse fibroblast L929 cells (ATCC\# CCL-1) were maintained at $37^{\circ} \mathrm{C}$ in a humidified atmosphere of $5 \% \mathrm{CO}_{2}$ in DMEM supplemented with $10 \% \mathrm{FBS}$ and $1 \%$ PSA (10000 unit/mL penicillin, $10 \mathrm{mg} / \mathrm{mL}$ streptomycin, $0.025 \mathrm{mg} / \mathrm{mL}$ amphotericin). All the cells in experiment were preceded within 10 passages. The pearl extract gel stock was prepared by dissolving powder in $\mathrm{PBS}$ at $\mathrm{pH}=7.2$. Cells were treated with different doses of pearl extract gel from 0 to $100 \mu \mathrm{g} / \mathrm{mL}$ and incubated at $37^{\circ} \mathrm{C}$.

\subsection{UVB irradiation to HaCaT and DNA damage quantification}

UVB irradiation was performed on the serum-starved monolayer cultures utilizing FS20 Lamp (New Vision Inc., Taiwan) instrument shown as Figure 1. The UVB source was a bank of two FS20 Lamps that emitted a continuous spectrum from 270 to $390 \mathrm{~nm}$, with a peak emission at $313 \mathrm{~nm}$; approximately $65 \%$ of the radiation is within the UVB $(280-320 \mathrm{~nm})$ wavelength.

Let the laser irradiated cells vertically upon the plate. The distance from the lamp to plate was $7 \mathrm{~cm}$. The energy output of UVB was measured by UVB photometer. Cells were seeded in 24-well plate at a

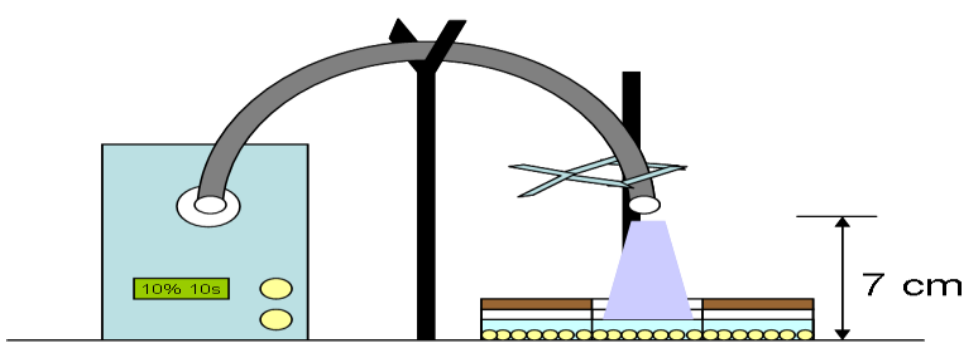

Fig. 1. UVB instrument setup. 
density of $1.5 \times 10^{5}$ cells/well for 4 hours before experiment. Then the supernatant was aspirated prior to irradiation and replaced with a thin layer of PBS to cover the cells. The cells were irradiated with UVB in a minimum amount of PBS (about $3 \mathrm{ml}$ ). The control cells were sham irradiated. After UVB irradiation, the cells were cultured in serum-free medium for the indicated time with or without the pearl extract and poly ( $\gamma$-glutamic acid) composite hydrogels.

Oxidative damage to DNA was a result of the interaction of DNA with reactive oxygen species (ROS). In particular, the hydroxy radical was converted from superoxide and hydrogen peroxide by the Fenton reaction. Assessing UVB irradiation-induced DNA damage was using Oxidative DNA Damage Kit from Kamiya Biomedical Company. DNA Damage Quantification Kit -AP Site Counting was made for the determination of abasic sites in sample DNA, which corresponded to colorimetric 96-well microplate assay.

\section{Assessment of cell viability (MTT assay)}

The cell viability was determined colorimetrically by the MTT reagent.

Cells were seeded in 96-well plate at a density of $3 \times 10^{4}$ cells/well and incubated for 4 hours prior to the cytotoxicity analysis of pearl extract and poly $(\gamma$-glutamic acid) composite hydrogels (pearl extract gel). After treatment, the supernatant was removed and subsequently added $0.5 \mathrm{mg} / \mathrm{mL}$ MTT solution and incubated at $37^{\circ} \mathrm{C}$ for 3 hours. After incubation, the supernatant was aspirated and cells were added with DMSO. The plate was placed on the orbital shaker for 10 minutes. The resulting absorbance from each well was measured at a wavelength of $540 \mathrm{~nm}$ with background subtraction at $650 \mathrm{~nm}$.

\subsection{Real-time PCR}

After treatments, total RNA was extracted using TRIzol reagent. The amount of each RNA sample was determined by Qubit fluorometer (Invitrogen). Reverse transcription was performed in a 20-L reaction system with 200 ng total RNA using high capacity cDNA reverse transcription kits. Relative quantification of inflammation mediator and apoptosis indicator (ex. IL-6, TNF-alpha and BCL2 etc.) were assessed by real-time PCR using ABI 7900HT (ABI) system. A housekeeping gene GAPDH was used as an internal control.

\subsection{Statistical analysis}

Statistical differences between experimental groups were determined by one-way analysis of variance (ANOVA).

All the data given represented the mean \pm standard error of the mean (SEM).

\section{Results}

\subsection{Effects of pearl extract gel on UVB-induced cytotoxicity in HaCaT}

Pearl extract gel caused a little toxicity on $\mathrm{HaCaT}$ cells at concentration exceeding $50 \mu \mathrm{g} / \mathrm{mL}$, whereas no cytotoxic effect on L929 cells was observed (Figure 2). 

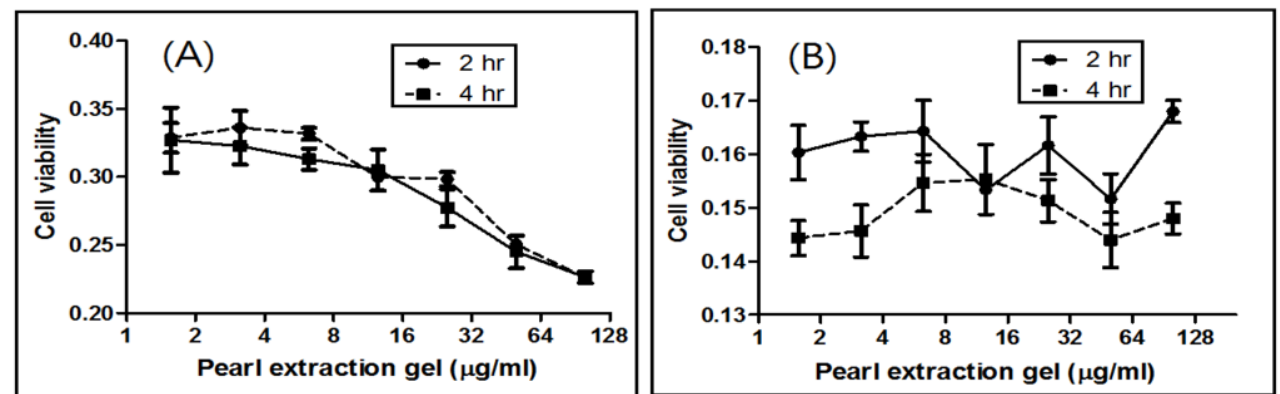

Fig. 2. (A) Effect of pearl extract gel on HaCaT cell viability. Cells were treated with a series of concentrations of pearl extract and poly ( $\gamma$-glutamic acid) composite hydrogels (pearl extract gel) for 2 and 4 hours. Cell viability was measured using MTT assay. (B) Effect of pearl extract gel on L929 cell viability. Cells were treated with a series of concentrations of pearl extract gel for 2 and 4 hours. Cell viability was measured using MTT assay.
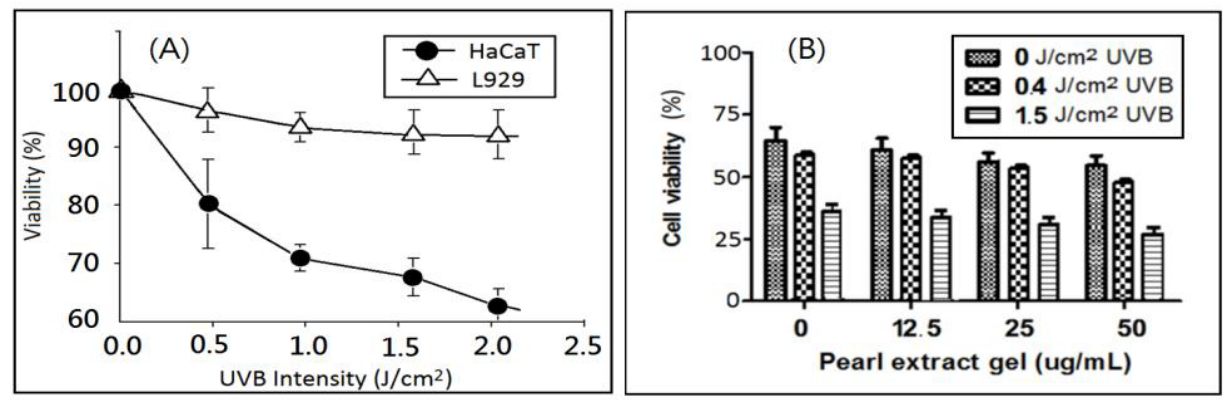

Fig. 3. (A) Effect of lower-intensity of UVB exposure on cell viability. HaCaT Cells were exposed to several dose of UVB irradiation. After 2 hours incubation with DMEM, cell viability was measured using MTT assay. L929 Cells were exposed to several dose of UVB irradiation. After 2 hours incubation with DMEM, cell viability was measured using MTT assay. (B) Effect of pearl extract gel on HaCaT cell exposed to UVB. HaCaT Cells exposed to UVB were treated with several doses of pearl extract gel for 24 hours. Cell viability was measured using MTT assay.

In order to assess the effect of the co-treatment of UVB and pearl extract gel on the cell cytotoxicity, the HaCaT cells were exposed to different dose of UVB $\left(0,0.4,1.5 \mathrm{~mJ} / \mathrm{cm}^{2}\right)$, or to UVB which was immediately followed by the addition of three doses of pearl extract $(12.5-50 \mu \mathrm{g} / \mathrm{mL})$ for the MTT assay. In the present study, treatment of the cells exposed to doses of UVB $\left(0.4 \mathrm{~mJ} / \mathrm{cm}^{2}\right)$ with $12.5-50$ $\mu \mathrm{g} / \mathrm{mL}$ of pearl extract gel resulted in weak cytotoxicity, treatment of the cells with UVB alone resulted in 38\% cytotoxicity. However, pearl extract gel treatment immediately after UVB exposure showed enhanced cell viability (37-62\%) (Figure 3 ).

\subsection{Anti-inflammation and anti-apoptosis effect on pearl extract gel}

It was hypothesized that pearl extract gel had a capability of compensating the adverse effect caused by UVB irradiation in the aspects of inflammation and apoptosis. HaCaT cells were exposed to UVB at a various density for 10 seconds, followed by treatments with pearl extract gel. Viabilities of cells that were irradiated and treated with pearl extract gel for treated for various hours were persistently decreased. Thus, UVB irradiation of $1.5 \mathrm{~J} / \mathrm{cm}^{2}$ which was the lowest power available was applied to the following experiments. Expressions of inflammation related genes in $\mathrm{HaCaT}$ cells after UVB exposure was increased significantly and pearl extract gel treatment resulted in insignificant suppression on the expressions (Figures 4A-4D). The inflammatory cytokine production of HaCaT 
cells exposed to UVB with or without pearl extract gel treatment was undetected. Regarding antiapoptotic effect of the pearl extract gel, BAX/Bcl2 ratio of UVB treated cells was found unaffected in presence of pearl extract gel (Figure 4E).

\section{Conclusion}

RTSES could provide a novel method for extracting the water soluble matrixes from pearl powder. It has been previously reported that pearl extract can protect keratinocyte cells from UVB-induced damage in vitro by acting as an antioxidant and a growth promoter. The significant cell death and viability of irradiated cells resulted from UVB irradiation are remarkably low in presence of pearl extract and poly ( $\gamma$-glutamic acid) composite hydrogels. Changes in expression of inflammation related genes between treatment and control groups are also significant. Difference in ratio of $\mathrm{BAX} / \mathrm{Bcl} 2$ between treatment and control groups is significant as well. These results suggest that pearl extract can activate rat keratinocyte cells and promote the anti-inflammation and anti-apoptosis effects on UVB irradiation $\mathrm{HaCaT}$ cells in vitro. Therefore, pearl extract and poly ( $\gamma$-glutamic acid) composite hydrogels may be effective in protecting skin against UVB. In conclusion, this study has established a simple in vitro model to screen active compounds from natural products related to wound healing.
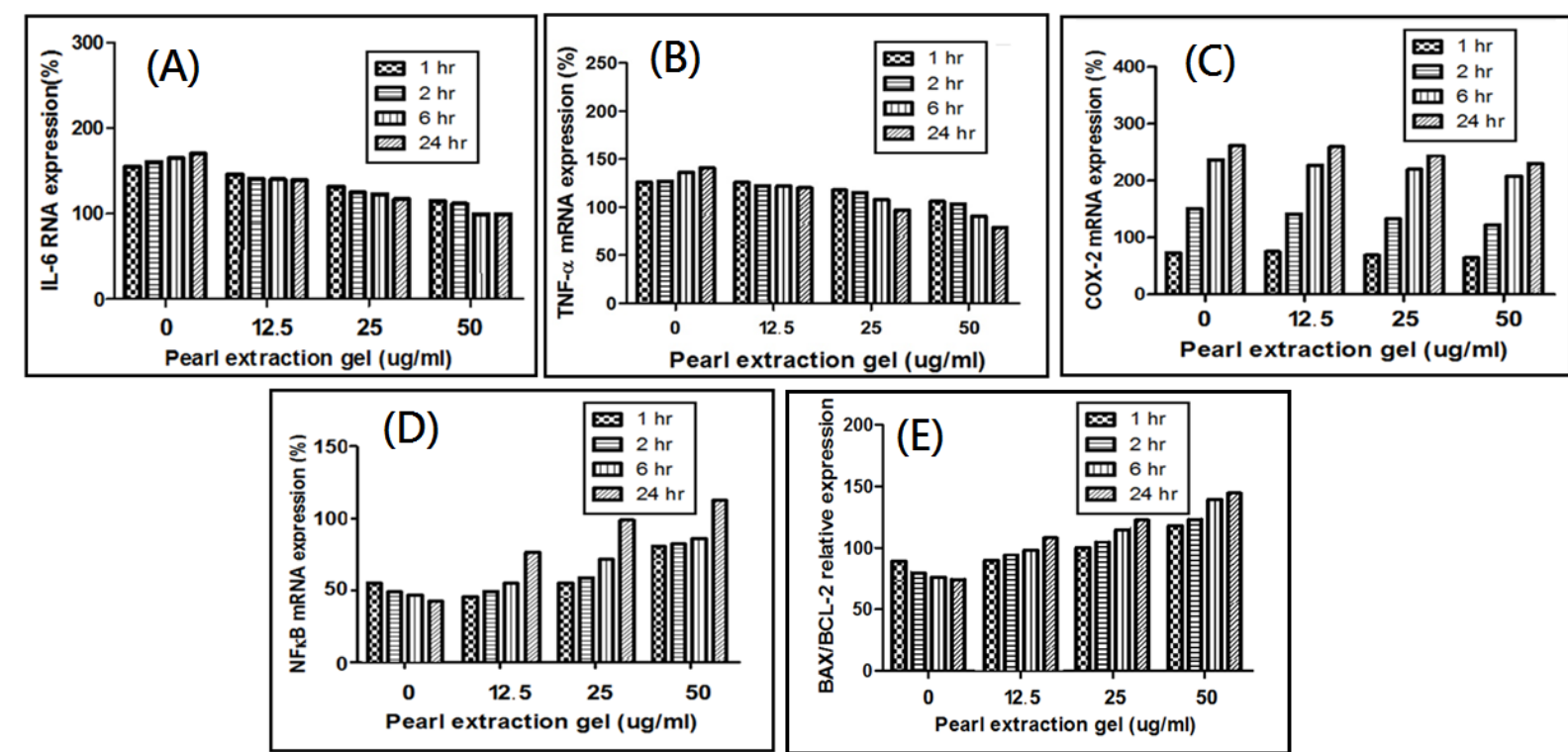

Fig. 4. (A )Effect of pearl extract gel on gene expression of IL-6, (B) Effect of pearl extract gel on gene expression of TNFalpha, (C) Effect of pearl extract gel on gene expression of COX-2, (D) Effect of pearl extract gel on gene expression of NFkappa $\mathrm{B}$ and (E)Effect of pearl extract gel on apoptosis-related gene expression. HaCaT Cells exposed to $1.5 \mathrm{~J} / \mathrm{cm} 2 \mathrm{UUVB}$ and the recovered with several doses of pearl extract gel for 1,2,6 and 24 hours. Total RNA was isolated from cells to perform real-time PCR. The transcriptional level of IL-6 was expressed as IL-6: GAPDH ratio $(\mathrm{p}<0.05)$. The transcriptional level of TNF-alpha was expressed as TNF-alpha: GAPDH ratio $(\mathrm{p}<0.05)$. The transcriptional level of COX-2 was expressed as COX-2: GAPDH ratio. The transcriptional level of NF-kappa B was expressed as NF-kappa B RNA: GAPDH ratio $(\mathrm{p}<0.05)$. The data was expressed as BAX: $\mathrm{Bcl} 2$ ratio $(\mathrm{p}<0.05)$. 


\section{Acknowledgments}

The authors would like to thank Chang Gung Memorial Hospital Grant (CMRPG290471) and Fu Jen Catholic University Grant (600090) for financially supporting this research. The RTSES pearl extract technical assistance of Mei-Wen Kao (MESOPHASE TECHNOLOGIES, INC, Taiwan) is gratefully acknowledged.

\section{References}

[1] J.P. Dai, Effects of pearl powder extract and its fractions on fibroblast function relevant to wound repair, Pharmaceutical Biology 48 (2010), 122-127.

[2] Y.C. Liu, K. Uchiyama and Y. Hasegawa, In vitro activities of the components from scallop shells, Fisheries Science $\mathbf{6 8}$ (2002), 1330-1336.

[3] Y.C. Liu, A. Torita and Y. Hasegawa, Scallop shell extract promotes recovery from UV-B-induced damage in rat skin epidermal layer, Fisheries Science 72 (2006), 388-392.

[4] S. Sudo, T. Kujikawa, T. Nagakura, T. Okubo, K. Sakaguchi, M. Tanaka, K. Nakashima and T. Takahashi, Structure of mollusk shell framework proteins, Nature 387 (1997), 563-564.

[5] T. Miyashita, R. Takagi, M. Okushima, S. Nakano, H. Miyamoto, E. Nishikawa and A. Matsushiro, Complementary DNA cloning and characterization of pearlin, a new class of matrix protein in the nacreous layer of oyster pearls, Marine Biotechnology 2 (2000), 409-418.

[6] T. Samata, N. Hayashi, M. Kono, K. Hasegawa, C. Horita and S. Akera, A new matrix protein family related to the nacreous layer formation of Pinctada fucata, FEBS Letters 462 (1999), 225-229.

[7] H.B. Xu, K.X Huang, Q.H. Gao, Z.H. Gao and X.X. Han, A study on the prevention and treatment of myopia with nacre on chicks, Pharmaceutical Research 44 (2001), 1-6.

[8] M.J. Almeida, L. Pereira, C. Milet, J. Haigle, M. Barbosa and E. Lopez, Comparative effects of nacre water-soluble matrix and dexamethasone on the alkaline phosphatase activity of MRC-5 fibroblasts, Journal of Biomedical Materials Research 57 (2001), 306-312.

[9] M.J. Almeida, C. Milet, J. Peduzzi, L. Pereira, J. Haigle, M. Barthelemy and E. Lopez, Effect of water-soluble matrix fraction extracted from the nacre of Pinctada maxima on the alkaline phosphatase activity of cultured fibroblasts, Journal of Experimental Zoology 288 (2000), 27-334.

[10] G. Cravotto, A. Binello, G. Merizzi and M. Avogadro, Improving solvent-free extraction of policosanol from rice bran by high-intensity ultrasound treatment, European Journal of Lipid Science and Technology 106 (2004), 147-151.

[11] C. Liu, S. Zhang and H. Wu, Non-thermal extraction of effective ingredients from Schisandra chinensis Baill and the antioxidant activity of its extract, Natural Product Research 23 (2009), 390-1401.

[12] S.R. Bae, C. Park, J.C. Choi, H. Poo, C.J. Kim and M.H. Sung, Effects of ultra high molecular weight poly-gammaglutamic acid from Bacillus subtilis (chungkookjang) on corneal wound healing, Journal of Microbiology and Biotechnology 20 (2010), 803-808. 